Brieger, E. M., Cosslett, V. E. \& Glauert, A. M. (1954). J. gen. Microbiol. 10, 294-303.

\title{
Reproductive Changes in Avian Tubercle Bacilli studied with the Electron Microscope
}

\author{
By E. M. BRIEGER, V. E. COSSLETT AND \\ AUDREY M. GLAUERT* \\ Papworth Hospital and the Cavendish and Strangeways Laboratories, \\ Cambridge
}

SUMMARY: The various stages of the reproductive cycle in freshly transplanted cultures of avian tubercle bacilli were recorded by a series of successive electron micrographs. An early stage of cytoplasmic differentiation is described in which discrete cellular units form inside the elongating filaments. Their significance and relation to the vacuolization seen at later stages is discussed. Evidence is produced to show that the dense bodies of mycobacteria are not nuclei. Free granules do not appear to play any part in the reproductive cycle in the normal type of development. In mycelial variants lysis is often seen to initiate new growth which starts from submicroscopical elements and micro-rods.

The electron microscope has already produced valuable information about the internal structure of tubercle bacilli in various stages of development. However, the results obtained still lack coherence and have given rise to conflicting interpretations of the cytological details observed. In particular there has been controversy concerning the nature of the dense spherical bodies which seem to be a characteristic of the species of Mycobacterium although they are also present in related species (Mudd, Brodie, Winterscheid, Hartman, Beutner \& McLean, 1951). They were first described by Lembke \& Ruska (1940) and have been variously identified as nuclei (Knaysi, Hillier \& Fabricant, 1950), nucleoids or karyoids (Ruska, Bringmann, Neckel \& Schuster, 1952), mitochondria (Mudd, Winterscheid, DeLamater \& Henderson, 1951), metachromatic granules (Werner, 1951) and skeletal structures for the support of nucleic acids, phosphates, lipoids, waxes, etc. (Lembke, 1947). Again there is considerable difference of opinion as to the nature of the intracellular organization seen at various stages of development (Brieger, Crowe \& Cosslett, 1947; Brieger \& Cosslett, 1949; Knaysi et al. 1950; Ruska et al. 1952).

The correlation of morphological and intracellular changes with stages in the reproductive process requires an almost continuous series of electron micrographs covering every phase of the development. The present paper describes results obtained by paying particular attention to the early stages of development of various avian strains grown on electron microscope grids placed on a variety of media.

\section{MATERIALS AND METHODS}

Six avian strains were investigated with the electron microscope. Of these two (Bone and Dobson) were highly virulent, two (AF and Lister) were avirulent and one (Fn) showed declining virulence. All except the Lister strain 
were freshly recovered from tuberculous birds. The present paper deals mainly with observations on the Fn strain which was compared with the virulent Sheard strain used by American workers.

\section{Technique}

In early work we used a stripping technique (Brieger et al. 1947) in which the bacilli were removed from a solid medium by covering it with a thin collodion membrane which was then floated off on water. Although giving a satisfactory picture of individual organisms this method suffers from the defect that an unknown proportion of the growth remains on the medium. Also, the amyl acetate used as a solvent in laying down the collodion film may induce changes in internal structure. Later a method was developed of growing bacilli directly on collodion-covered electron microscope grids (Brieger \& Cosslett, 1949) and this has recently been modified for use with liquid media.

Stainless steel electron microscope grids were used, as it was suspected that copper grids would have an inhibitory effect on the growth of the bacilli. The grids were sterilized and covered with a thin formvar membrane in the usual way; formvar was chosen instead of collodion because of its greater strength. Sterile filtered water was used for the preparation of the films to eliminate the contaminating organisms that are invariably present in distilled water.

A thin layer of cotton-wool was placed at the bottom of a small Petri dish $(5 \mathrm{~cm}$. diameter, $2 \mathrm{~cm}$. deep) and covered with a disk of filter-paper. After sterilization the cotton-wool was thoroughly soaked with the fluid medium and up to 20 grids placed on the filter-paper with the formvar films uppermost. Each grid was then inoculated with a drop of bacillary suspension in distilled water by means of a fine platinum loop $1 \mathrm{~mm}$. in diameter. If the drop did not stay on the surface of a grid, it was assumed that the holes in the formvar film were too large and the grid was discarded. After the desired time of growth each grid was removed, floated on sterile distilled water to remove any medium adhering to the lower surface, fixed by floating on $5 \%$ formalin for $30 \mathrm{~min}$., and washed again to remove any excess fixative. Care was taken throughout the procedure that the inoculated surface of the grid should remain undisturbed. In dealing with pathogenic organisms this method is preferable to that of Hillier, Knaysi \& Baker (1948) in which bacilli are grown on a collodion film which is later floated off the medium and mounted on grids.

A preliminary experiment was carried out to show that sufficient nutrient medium diffuses through the formvar film. The growth on the grids was compared with growth directly on the surface of a solid medium, by taking impression prints on clean formvar-covered grids from the growth on a Löwenstein plate. The comparison showed that with Dubos medium the fact that the nutrient fluid had to diffuse through the formvar film did not impair growth to any noticeable extent. A solid medium can be used if a film of saline solution is introduced between the medium and the grid to prevent the formation of an air gap.

Before examination in the electron microscope the grids were viewed with 
the $\times 40$ dry objective of a phase-contrast microscope so that a comparison of electron and phase-contrast pictures could be made. However, the stainless steel electron microscope grids used for the growth experiments consist of a mesh of woven steel wires and are not flat, so that it was difficult to obtain good phase-contrast photographs. When taking impression prints the flatter copper grids were used, and it was with these that the best phase-contrast photographs were obtained. In future we plan to use flat silver-plated copper grids for the growth experiments.

\section{RESULTS}

\section{The reproductive cycle}

The general outline of the standard development in micro-cultures of avian tubercle bacilli was known from recent work with the light microscope (Brieger \& Fell, 1946), stained preparations (Bretey \& Imelik, 1949) and the phase-contrast microscope (Brieger \& Glauert, 1952). When a young culture is first transplanted to a fresh medium it consists mainly of short coccoid rods. These elongate into filaments $(8-10 \mu$.) which continue to divide and grow during a phase of filamentous proliferation. After this phase the filaments suddenly break down into masses of short rods which elongate to form the new generation and the cycle is complete. The examination of inoculated grids with the phase-contrast microscope revealed that a similar cycle takes place on the grids, and the observations provide a useful basis for the interpretation of the corresponding electron micrograms. At any one time different parts of the same micro-culture are in different stages of development and, similarly, a variation is also noticeable between one grid square and another. However, the general pattern of growth is uniform throughout, and electron micrographs have been chosen to illustrate every important phase in the development.

The original inoculum. The original suspension was usually taken from a 2- to 3-week old culture growing on Löwenstein's medium, and the inoculum then consisted mainly of well-defined coccoid rods which lay singly or in groups (Pl. 1, fig. 1). The rods varied in length from 0.5 to $1.0 \mu$. and usually each had two dense raised polar bodies and a uniformly transparent cytoplasm. Evidence for the multiplication of these rods by simple fission was never observed.

Elongation of rods into filaments. During the first 20-24 hr. of incubation the rods of the inoculum began to elongate into filaments of $2-4 \mu$. in length (Pl. 1, fig. 2a-d). In most of the filaments the cytoplasm was seen to be differentiating into a series of separate cellular units each surrounded by a definite limiting membrane. In some filaments the units appeared very dense (Pl. 1, fig. 2c) and even caused a swelling of the filament. Sometimes the units formed two groups which retracted away from each other towards the ends of the bacillus (Pl. 1, fig. 3) leaving a comparatively transparent central region. The outer membrane of the whole cell remained unbroken and the filament did not immediately divide. The filaments usually have two fully developed dense bodies in polar positions and in some organisms a number of smaller ones are 
also seen scattered among the cellular units and apparently associated with them (Pl. 1, fig. 2d).

Stage of filamentous proliferation. After the first filaments were formed a rapid proliferation of filaments occured until the grid squares were covered with organisms. The filaments grew and subdivided, frequently remaining attached to each other (Pl. 1, fig. 4). Sometimes they had a characteristic mycelial appearance in phase-contrast photographs (Pl. 2, fig. 5), but when a selected group of organisms from the same grid square was viewed in the electron microscope (Pl. 2, fig. 6) it was seen that the filaments were quite separate, that there was no true branching and that the mycelial appearance was produced because the filaments often remained stuck together. Each daughter filament contained rows of the intracellular units and a number of dense bodies, which in stages of rapid growth were often very small.

The new generation of rods. The final stage in the reproductive cycle led to a massive production of small rods. In the phase-contrast microscope the spaces between the filaments were found to be packed with transparent rods which appeared to have developed from the filaments (Pl. 2, fig. 7). The change occurred very rapidly, and as a result there was no definite evidence in electron micrographs of how the transition from filaments to rods occurred. Sometimes transverse division lines were seen but they were not present in sufficient numbers to indicate that transverse fission was the only means of division. Pl. 2, fig. 8, shows that other processes such as division by retraction or liberation of intracellular units must be considered.

When the cycle was complete, electron micrographs showed masses of rods of fairly uniform size (Pl. 2, fig. 9). Each bacillus usually contained two dense polar bodies, but otherwise the cytoplasm showed little structure and had the uniform appearance seen in the rods of the original inoculum. There are many variations of this cycle depending upon the age of the original culture, the strain and the medium used, but the basic pattern is the same.

\section{Vacuolization}

In early observations made with the stripping technique an internal organization was seen in the filaments which consisted of rows of definite units with transparent centres (Pl. 3, fig. 10). At first it was thought that the transparency was due to the solvent action of the amyl acetate used in forming the collodion film, but in later investigations with other techniques similar vacuolized structures were found ( $\mathrm{Pl}$. 3, fig. $11 a, b$ ) in the stage of filamentous proliferation. These vacuolized units had a similar location to the intracellular units and in fact all shades of density of the units were observed (Pl. 3, fig. $11 a$ ). In some cultures the vacuolized units were so numerous that the filaments were packed with transparent bodies each with its limiting membrane ( $\mathrm{Pl} .3$, fig. 12). This vacuolization was seen where conditions were apparently not favourable for normal growth and filaments persist.

\section{Dense bodies}

Accompanying the excessive vacuolization of the filaments there was frequently an unusual development of the dense bodies which sometimes grew 
to giant sizes and filled considerable sections of the filaments (Pl. 3, fig. 13). As a result of even light electron bombardment the bodies were sometimes differentiated into a dark cortex and lighter core (Pl. 3, fig. 14). A similar structure was found in the dense bodies of Mycobacterium phle $i$ which sometimes contained two or three lighter areas (Pl. 4, fig. 15).

When growth was very rapid the final rods sometimes had no dense bodies or only very small ones, and a striking arrangement of minute dense bodies was sometimes seen (Pl. 4, fig. 16). Alternatively, the dense bodies outnumbered the filaments which became transparent and the whole field had a spotted appearance (Pl. 4, fig. 17). In such pictures the impression is produced that dense bodies are liberated from the filaments.

\section{Mycelial type of growth}

(a) When the original inoculum was taken from an old culture which has been growing on Löwenstein's medium for some months it consisted of rods which had merged into clumps (Pl. 4, fig. 18a,b) and some cells appeared as empty sheaths with no solid content.

The subsequent development of these forms differed from that in young cultures and many amorphous structures were found which contained electronscattering bodies of various sizes and shapes (Pl. 4, fig. 19). New growth appeared to originate in faint mycelial networks in which pear-shaped structures predominated ( $\mathrm{Pl}$. 4, fig. 20). These structures resembled the intracellular units and often had a pair of small dense bodies.

(b) In cultures which had wholly reverted to the mycelial type of growth large branched structures were seen which sometimes covered several squares of the grid, and electron micrographs showed that true branching and budding occurred (Pl. 5, fig. 21). The mycelial branches were usually transparent to the electron beam, and there was no evidence of a definite organization similar to that seen in normal filaments. It appears that lateral buds are formed which later grow into rods and then break off. Division also occurred by transverse fission after the appearance of irregularly spaced division lines and the rounding up of the newly formed ends (Pl. 5, fig. 22).

A process of 'lysis' was often seen in mycelial cultures in which the filaments swelled, became less distinct and were surrounded by rods embedded in amorphous and granular material (Pl. 5, fig. 23). In later stages the filaments apparently released large numbers of short bipolar rods, some of which were as small as $0 \cdot 4 \mu$. (Pl. 5, fig. 24). From phase-contrast studies (Brieger \& Glauert, 1952 ) it is known that this material may be the basis of new growth, but further work is necessary to observe parallel developments in electron micrographs.

\section{Influence of medium}

The medium and technique used influenced the type and rate of the resultant growth. Abnormal forms were most common in the later stages of growth on solid media, particularly when there was a formvar film between the culture and the medium. In development on Dubos medium on grids distorted forms were rare. Embryo extract diffuses poorly through the formvar film so 
that growth is much retarded, and in order to obtain normal growth the grids must be inverted and placed with the inoculated surfaces in contact with the medium. The cytological changes were similar whatever media were used, but the intracellular units were most evident in impression prints taken from growth on Löwenstein's medium.

Comparison of strains. The virulent Sheard strain showed similar developments to the Fn strain, and throughout the experiments there was no obvious difference between virulent and avirulent strains. Consequently, electron micrographs cannot be used as a basis for strain identification.

\section{DISCUSSION}

The results presented in this paper provide new evidence of the cytological changes accompanying the reproduction of avian tubercle bacilli. In particular, a striking intracellular organization of the bacilli is described which was not observed by previous workers. It was most obvious during the first 20-30 hr. of incubation when rapid elongation was occurring, and escaped the notice of Ruska et al. (1952) who only began their observations after $48 \mathrm{hr}$. Knaysi et al. (1950) show an electron micrograph taken after $21.5 \mathrm{hr}$. incubation in which the filaments are already highly vacuolized and there is no sign of the intracellular units which in our view precede the vacuolization. Knaysi apparently obtained his material from a fluid medium, in which filaments persist. In order to observe the early phases of growth the material must be removed from a culture which has been growing for no more than 2-3 weeks on a solid medium, when coccoid rods predominate.

The formation of cellular units within the bacillary filaments seems to support the view that mycobacteria are multicellular organisms (Bisset, 1950). However, this organization is only a passing stage coinciding with the multinuclear organization revealed by the $\mathrm{HCl}+$ Giemsa method (Brieger \& Robinow, 1947). So far as is known there is no parallel development in the bacteria but in the nocardia, which are more closely related to the mycobacteria than is generally believed (Brieger \& Glauert, 1952), some sort of analogy can be found in the description of spore formation. 'Spores are formed by the breaking up of the cell plasm into separate portions, usually $\mathbf{3}$ to $\mathbf{5}$ in number. Every portion becomes rounded, covered with a membrane, and transforms into a spore; the membrane of the mother cell dissolves and disappears' (Waksman, 1950). Spore formation in the mycobacteria has often been suspected since it was first suggested by Robert Koch, but the idea has been generally discarded. The fact that these units are often associated with a single dot or micro-form of the dense spherical granule seems to support the view that the units represent rods which are being pre-formed inside the filaments (Gróh, 1933). The formation of rods within filaments is a common occurrence in mycelial variants of avian strains (Brieger \& Glauert, 1952).

A difficulty arises in relating this intracellular organization to the vacuolization seen at a still later stage. Knaysi et al. (1950) stated that these vacuoles 'vary considerably in size, 500 to $3000 \mathrm{~A}, \ldots$ are unstainable, highly transparent to the electron beam, exert turgor pressure, and accumulate solid content 
that tends to disappear in old cultures'. From these observations they identified them as 'sap-vacuoles'. The question arises whether the intracellular units and these vacuoles are independent structures or whether they are the same structures in different stages of development. Our observations show that transitional stages exist in which filaments contain intracellular units which have lost electron density and appear as transparent areas (Pl. 3, fig. 11 a). It thus seems possible that the vacuoles represent intracellular units which have become transparent as a result of chemical changes.

Having identified these structures as 'sap-vacuoles', Knaysi et al. (1950) concluded that the dense polar bodies were the nuclei. Ruska et al. (1952) also called them nucleoids or karyoids. However, this interpretation conflicts with the view of Robinow \& Cosslett (1948) that in bacteria the nuclei are electron transparent. Also Mudd, Winterscheid, DeLamater \& Henderson (1951) showed that the dense bodies gave a staining reaction characteristic of mitochondria, and the presence of nuclear structures between the dense bodies has also been demonstrated by means of the Feulgen stain (DeLamater, Hunter \& Mudd, 1952). From our observations it would certainly seem more likely that if discrete nuclei exist they should be found between the dense bodies at the site of the newly forming intracellular units.

The function of the dense bodies is still under discussion and their mode of multiplication is also obscure. There can be no doubt that they reproduce rapidly. There is evidence of multiplication by division (Knaysi et al. 1950), but one would expect to find many more instances of this division if it were the only means of reproduction (Werner, 1951). A second possibility is that the larger dense bodies fragment into smaller micro-forms, and a third that small dense bodies are synthesized in the cytoplasm (Lembke, 1947), although it is hard to imagine a satisfactory proof of this. Ruska et al. (1952) described how the dense bodies may grow to abnormal sizes in old cultures, and we have observed similar forms (Pl. 3, fig. 13). However, this is rarely seen in the course of normal development, and it seems more likely that this swelling is another sign of the arrest of normal growth, comparable to the excessive vacuolization that is also seen. Werner (1951) believed that in some strains dense bodies may be absorbed as growth reaches its peak; however, this appearance may be due to the rapid reproduction of rods without an accompanying multiplication of dense bodies, with the result that young rods may have only one polar body or two extremely small ones (Pl. 2, fig. 9).

Lembke (1947) showed that dense bodies may be spontaneously liberated from old cultures or produced by mechanical disintegration, but they were not viable under these conditions. Some of our electron micrographs also suggest that dense bodies are liberated (Pl. 4, fig. 17). In mycelial cultures lysis is frequently accompanied by the appearance of granular forms. Microrods containing two dense bodies (Pl. 5, fig. 24) are also seen associated with disintegrating mycelial filaments, and there is evidence from phase-contrast studies (Brieger \& Glauert, 1952) that these elements can germinate on removal to a fresh medium and produce a new generation of rods. 
We wish to express our thanks to Mr R. W. Horne and Mr H. E. Pearson for their help in taking the electron micrographs. We are also grateful to Dr Dobson, of the Ministry of Agriculture's Research Station, Weybridge, for supplying the material from which the avian strains were recovered. Dr Steenken of the Trudeau Laboratory, Saranac Lake, kindly supplied us with the Sheard strain.

\section{REFERENCES}

BIssetT, K. A. (1950). The Cytology and Life-History of Bacteria. Edinburgh: E. and S. Livingstone, Ltd.

Bretey, J. \& Imelik, S. (1949). Étude du mode de multiplication du bacille tuberculeux aviare (Mycobacterium tuberculosis) dans le milieu de culture de Dubos. Ann. Inst. Pasteur, 77, 228.

Brieger, E. M. \& Cosslett, V. E. (1949). Preparation of bacteria for electron microscopy. Nature, Lond. 164, 352.

Brieger, E. M., Crowe, G. R. \& Cosslett, V. E. (1947). Electron microscopy of bacteria. Nature, Lond. 160, 864.

Brieger, E. M. \& Fell, H. B. (1946). Warm-stage observations on the initial development of the avian tubercle bacillus cultivated in embryo extract. J. Hyg., Camb. 44, 158.

Brieger, E. M. \& Glauert, A. M. (1952). A phase-contrast study of reproduction in mycelial strains of avian tubercle bacilli. J. gen. Microbiol. 7, 287.

Brieger, E. M. \& RoBinow, C. F. (1947). Demonstration of chromatinic structures in avian tubercle bacilli in the early stages of development. J. Hyg., Camb. 45, 413.

Delamater, E. D., Hunter, M. E. \& Mudd, S. (1952). Current status of the bacterial nucleus, Exp. Cell Res., Suppl. 2, 319.

GróH, E. (1933). Über die Körnchen und Entwicklung des Tuberkuloseerregers. Zbl. Bakt. (1. Abt. Orig.), 128, 353.

Hillier, J., KNaYsi, G. \& Baker, R. F. (1948). New preparation techniques for the electron microscopy of bacteria. J. Bact. 56, 569.

Knaysi, G., Hillier, J. \& Fabricant, C. (1950). The cytology of an avian strain of Mycobacterium tuberculosis studied with the electron and light microscopes. J. Bact. 60, 423.

Lembke, A. (1947). Untersuchungen an den Frregern der Tuberkulose. Zbl. Bakt. (1. Abt. Orig.), 152, 239.

Lembke, A. \& Ruska, H. (1940). Vergleichende mikroskopische und übermikroskopische Beobachtungen an den Erregern der Tuberkulose. Klin. Wschr. 19, 217.

Mudd, S., Brodie, A. F., Winterscheid, L. C., Hartman, P. E., Beutner, E. H. \& MCLEAN, R. A. (1951). Further evidence of the existence of mitochondria in bacteria. J. Bact. 62, 729.

Mudd, S., Winterscheid, L. C., Delamater, E. D. \& Henderson, H. J. (1951). Evidence suggesting that the granules of mycobacteria are mitochondria. J. Bact. 62, 459.

Robinow, C. F. \& Cosslett, V. E. (1948). Nuclei and other structures of bacteria. J. appl. Physics, 19, 124.

Ruska, H., Bringmann, G., Neckel, I. \& Schuster, G. (1952). Über die Entwicklung sowie den morphologischen und zytochemischen Aufbau von Mycobacterium avium (Chester). Z. wiss. Mikr. 60, 425.

Waksman, S. A. (1950). The Actinomycetes. Waltham, Mass., U.S.A.: Chronica Botanica Co.

WERNER, G. H. (1951). Electron microscopic study on the cellular morphology of tubercle bacilli. Advanc. Tuberc. Res. 4, 53. 


\section{EXPLANATION OF PLATES}

\section{Plate 1}

Micro-cultures of Mycobacterium avium photographed with a Siemen's electron microscope operating at $70 \mathrm{kV}$. Some preparations shadowed at $30^{\circ}$ with gold-palladium.

Fig. 1. Starting preparation from an 18-day old culture. Short rods with dense polar bodies and structureless cytoplasm. Bone strain, shadowed, $\times 12,500$.

Fig. 2a-d. Impression prints taken after $20 \mathrm{hr}$. ( $a$ and $b$ ) or $48 \mathrm{hr}$. ( $c$ and $d$ ) growth on Löwenstein's medium. Discrete cellular units within elongating filaments. $(a)$ Fn strain, $\times 13,000$. (b) Fn strain, $\times 12,100$. (c) Fn strain, shadowed, $\times 11,200$. (d) Sheard strain, $\times 13,000$.

Fig. 3. Impression print taken after 2 days growth on Löwenstein's medium. Retraction of cellular units. Fn strain, shadowed, $\times 12,800$.

Fig. 4. 3 days growth on electron microscope grid on Löwenstein's medium. Filament segmenting. Dobson strain, shadowed, $\times 17,000$.

\section{Plate 2}

Comparative phase-contrast and electron photographs of avian tubercle bacilli in impression prints from Löwenstein's medium.

Fig. 5. $20 \mathrm{hr}$. Apparently mycelial arrangement of filaments. Sheard strain. Phasecontrast microscope, $\times 1500$.

Fig. 6. Electron microgram of group of filaments indicated in fig. 5. Electron microscope, $\times 12,100$.

Fig. 7. $40 \mathrm{hr}$. Massive production of rods from filaments. Sheard strain. Phase-contrast microscope, $\times 4000$.

Fig. 8. $20 \mathrm{hr}$. Corresponding phase in electron microscope. Sheard strain, $\times 12,600$.

Fig. 9. 8 days. Completion of cycle. New generation of rods. Fn strain. Electron microscope. Shadowed, $\times 12,400$.

\section{Plate 3}

Electron micrograms of avian tubercle bacilli showing vacuolization of the filaments and variations in dense bodies.

Fig. 10. $48 \mathrm{hr}$. growth on Löwenstein's medium removed by stripping with collodion film. Vacuolized filaments. AF strain, $\times \mathbf{2 2 , 0 0 0}$.

Fig. 11 $a$. Impression print from 4 days growth on Löwenstein's medium. Vacuolized filaments. Fn strain, shadowed, $\times 12,400$.

Fig. $11 b .8$ days growth on electron microscope grid on Löwenstein's medium. Vacuolized filaments. Fn strain, shadowed, $\times 12,600$.

Fig. 12. Impression print from 6 days growth on Löwenstein's medium. Filaments packed with vacuolized units. $\times \mathbf{1 2 , 4 0 0}$.

Fig. 13. 14 days growth on electron microscope grid on Löwenstein's medium. Swollen dense bodies. Fn strain, $\times 12,400$.

Fig. 14. 14 days growth on electron microseope grid on Löwenstein's medium. Vacuolated dense bodies. Fn strain, shadowed, $\times 12,500$.

\section{Plate 4}

Electron micrograms showing variations in dense bodies

Fig. 15. $70 \mathrm{hr}$. growth of Mycobacterium phlei on electron microscope grid on embryo extract. Dense bodies with lighter areas. Shadowed, $\times 12,500$.

Fig. 16. 14 days growth of avian tubercle bacilli on electron microscope grid on Löwenstein's medium. Micro forms of dense bodies in proliferating filaments. Fn strain, $\times 17,000$.

Fig. 17. 8 days growth of avian tubercle bacilli on electron microscope grid on Löwenstein's medium. Masses of dense bodies in transparent filaments. Fn strain, $\times 12,300$. 
Journal of General Microbiology, Vol. 10, No. 2
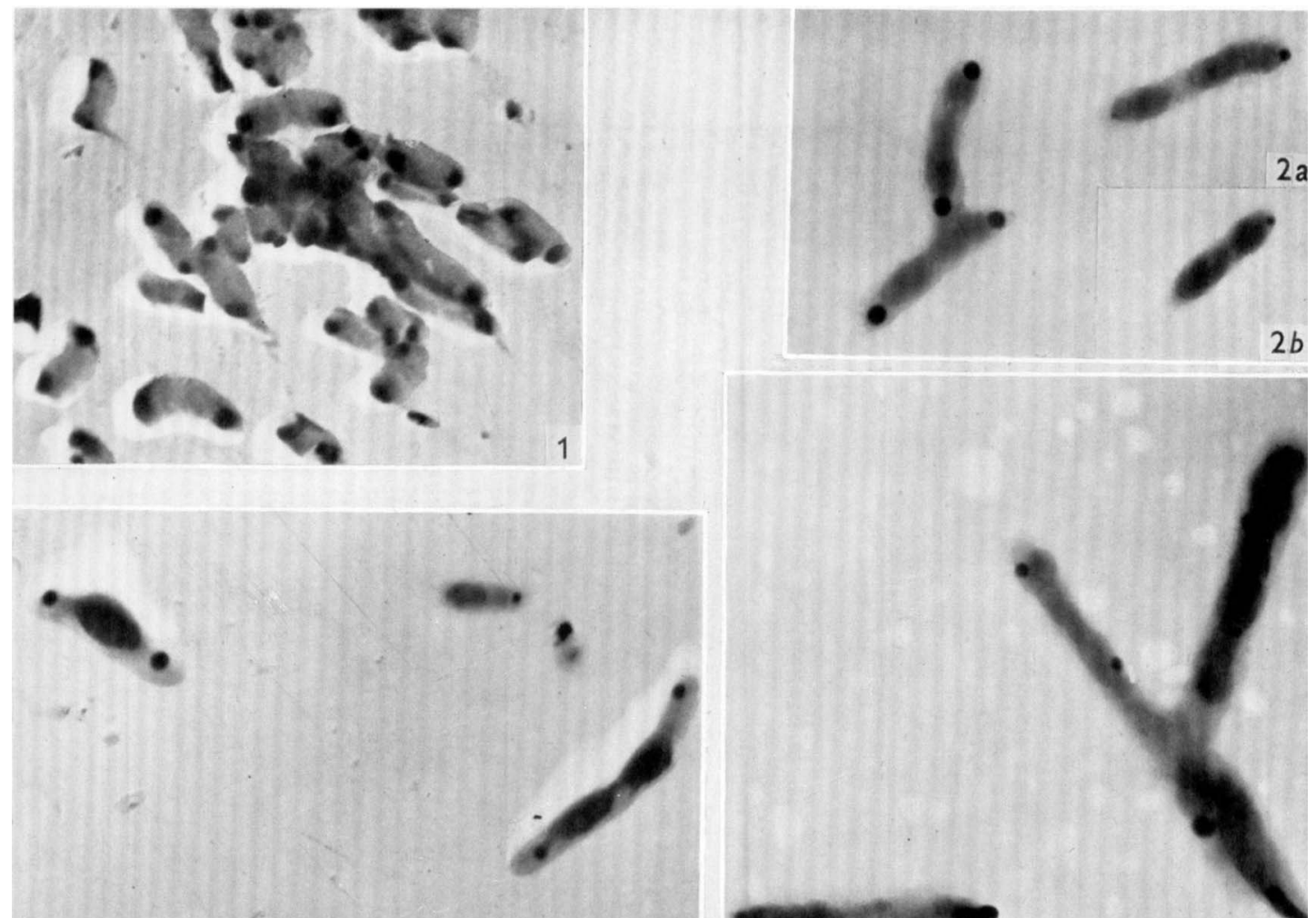

$2 c$
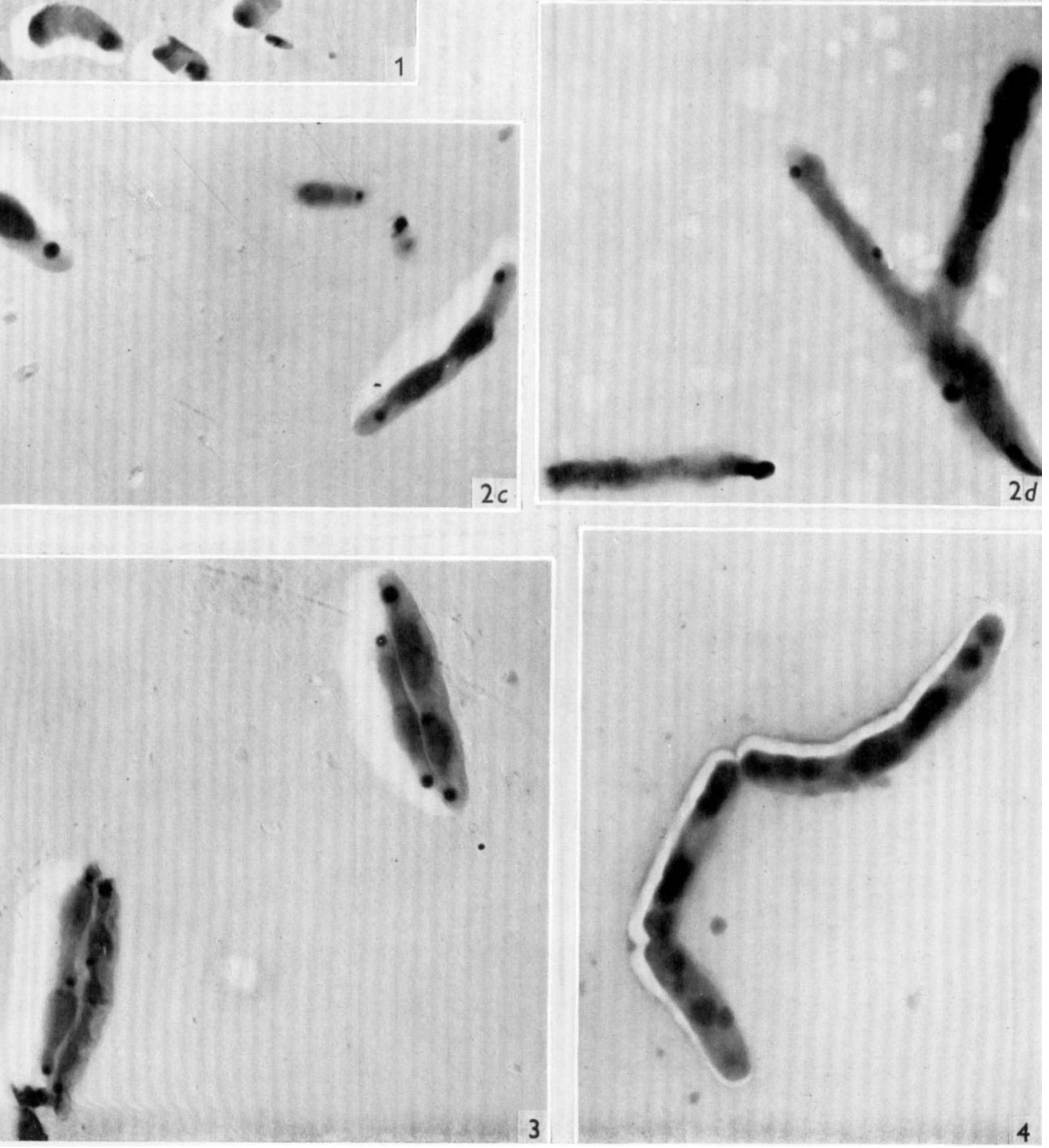

E. M. Brieger, V. E. Cosslett \& A. M. Glauert-Electron microscopy of avian tuberche Bacilli. Plate 1 
Journal of General Microbiology, Vol. 10, No. 2
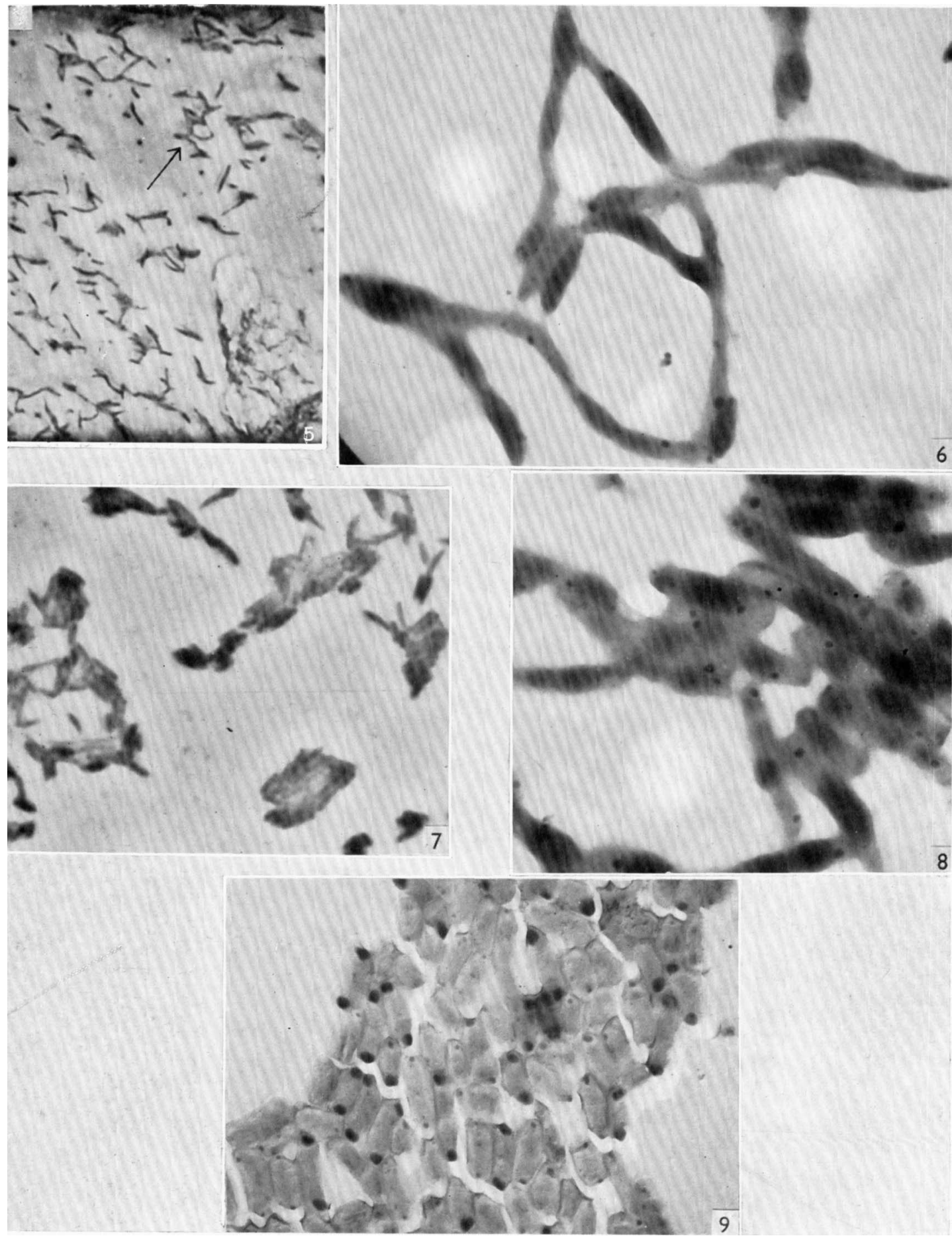

E. M. Brieger, V. E. Cosslett \& A. M. Glauert--Electron microscopy of avian tubercle bacilli. Plate 2 
Journal of General Microbiology, Vol. 10, No. 2
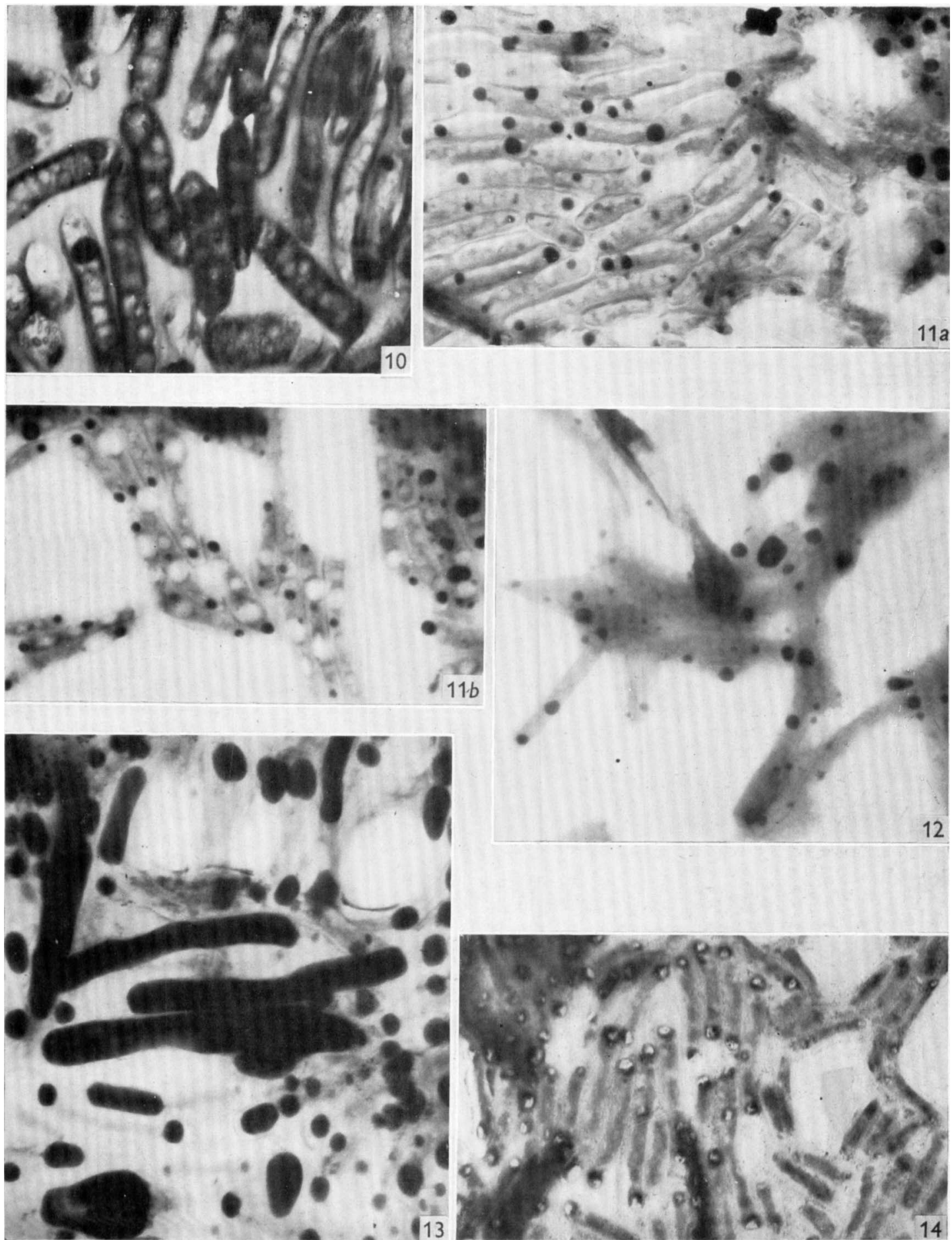

E. M. Brieger, V. E. Cosslett \& A. M. Glavert-Electron microscopy of avian tubercle Bacilli. Plate 3 
Journal of General Microbiology, Vol. 10, No. 2

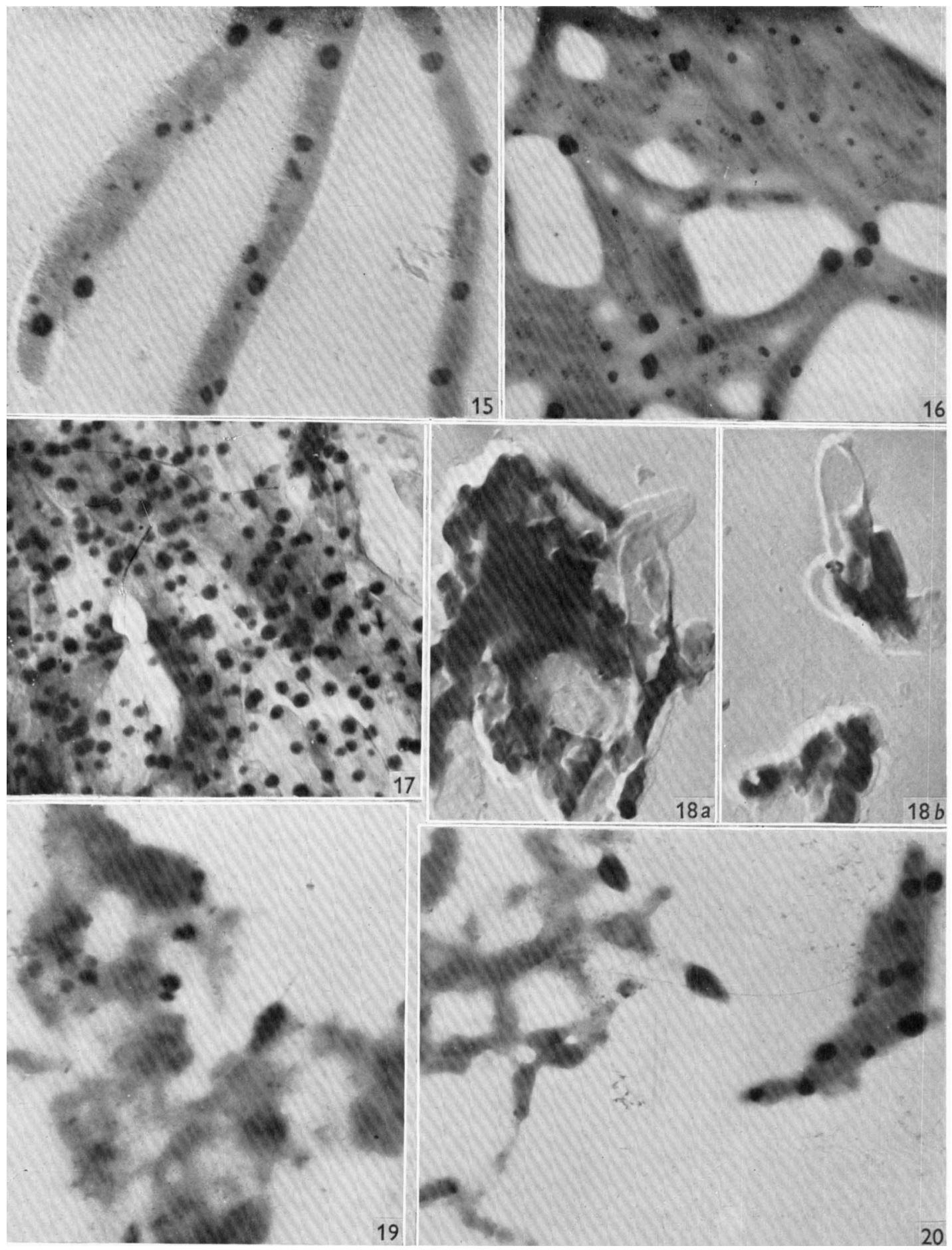

E. M. Brieger, V. E. Cosslett \& A. M. Glauert--Electron microscopy of avian tubercle bacilli. Plate 4 
Journal of General Microbiology, Vol. 10, No. 2
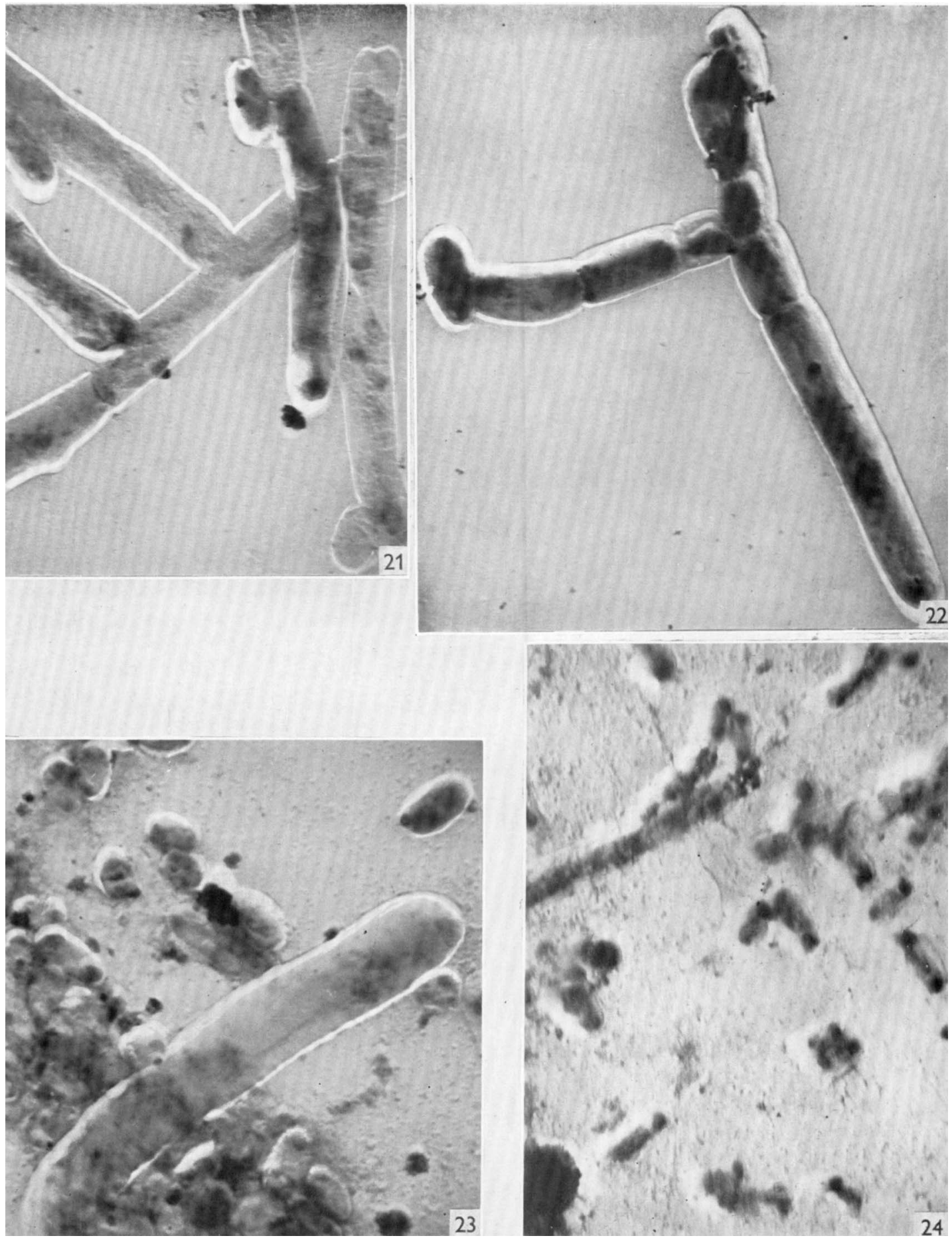

E. M. Brieger, V. E. Cosslett \& A. M. Glaulert-Electron microscopy of avian tubercle Bacilli. Plate 5 
Electron micrograms of development in old cultures of avian tubercle bacilli.

Fig. 18a, $b$. Starting preparation of 13-month old culture. Rods merged in clumps and empty sheaths. Fn strain, shadowed, $\times 13,000$.

Fig. 19. 2 days growth on electron microseope grid on Löwenstein's medium. Amorphous clump containing dense bodies. Fn strain, $\times 12,600$.

Fig. 20. 4 days growth on electron microscope grid on Löwenstein's medium. Pear-shaped

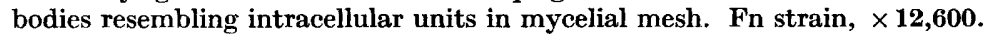

\section{Plate 5}

Electron micrograms of mycelial forms of avian tubercle bacilli. Material taken from a 7-week old culture of the Fn strain.

Fig. 21. Mycelium with transparent branches and associated micro-rods. Shadowed, $\times 18,000$.

Fig. 22. Segmenting, branched filament. Shadowed, $\times 20,000$.

Fig. 23. Lysis of mycelial filament. Associated granular elements. Shadowed, $\times 15,000$.

Fig. 24. Disintegrating mycelial filament and bipolar micro-rods. Shadowed, $\times 12,000$.

(Received 7 September 1953) 